\title{
A Transcription Elongation Factor That Links Signals from the Reproductive System to Lifespan Extension in Caenorhabditis elegans
}

\author{
Arjumand Ghazi, Sivan Henis-Korenblit, Cynthia Kenyon*
}

Department of Biochemistry and Biophysics, University of California San Francisco, San Francisco, California, United States of America

\begin{abstract}
In Caenorhabditis elegans and Drosophila melanogaster, the aging of the soma is influenced by the germline. When germline-stem cells are removed, aging slows and lifespan is increased. The mechanism by which somatic tissues respond to loss of the germline is not well-understood. Surprisingly, we have found that a predicted transcription elongation factor, TCER-1, plays a key role in this process. TCER-1 is required for loss of the germ cells to increase $C$. elegans' lifespan, and it acts as a regulatory switch in the pathway. When the germ cells are removed, the levels of TCER-1 rise in somatic tissues. This increase is sufficient to trigger key downstream events, as overexpression of tcer-1 extends the lifespan of normal animals that have an intact reproductive system. Our findings suggest that TCER-1 extends lifespan by promoting the expression of a set of genes regulated by the conserved, life-extending transcription factor DAF-16/FOXO. Interestingly, TCER-1 is not required for DAF-16/FOXO to extend lifespan in animals with reduced insulin/IGF-1 signaling. Thus, TCER-1 specifically links the activity of a broadly deployed transcription factor, DAF-16/FOXO, to longevity signals from reproductive tissues.
\end{abstract}

Citation: Ghazi A, Henis-Korenblit S, Kenyon C (2009) A Transcription Elongation Factor That Links Signals from the Reproductive System to Lifespan Extension in Caenorhabditis elegans. PLoS Genet 5(9): e1000639. doi:10.1371/journal.pgen.1000639

Editor: Stuart K. Kim, Stanford University Medical Center, United States of America

Received June 24, 2009; Accepted August 10, 2009; Published September 11, 2009

Copyright: (c) 2009 Ghazi et al. This is an open-access article distributed under the terms of the Creative Commons Attribution License, which permits unrestricted use, distribution, and reproduction in any medium, provided the original author and source are credited.

Funding: AG was supported by the Larry Hillblom Foundation for Aging Research postdoctoral grant. SHK was supported by a Human Frontier Science Program fellowship. This work was funded by National Institute of Health grant RO1 AG020932 to CK, who is an American Cancer Society Research Professor, director of University of California San Francisco's Hillblom Center for the Biology of Aging, and a founder and director of Elixir Pharmaceuticals. The funders had no role in study design, data collection and analysis, decision to publish, or preparation of the manuscript.

Competing Interests: The authors have declared that no competing interests exist.

*E-mail: cynthia.kenyon@ucsf.edu

\section{Introduction}

When the germline of $C$. elegans is removed, either by laser ablation or by mutations that block germline-stem cell proliferation, the animals live approximately $60 \%$ longer than normal $[1,2]$. This longevity is not a simple consequence of sterility, as removing the entire gonad (the germ cells as well as the somatic reproductive tissues) does not extend lifespan [1]. These and other findings suggest that both the germline cells and the somatic reproductive tissues influence lifespan [3,4]. A link between reproductive tissues and aging may be widespread in nature, as removing germline-stem cells during adulthood increases the lifespan of flies [5], as well as worms, and transplanting ovaries of young mice into older animals can increase lifespan as well [6].

The mechanism by which loss of the germline extends lifespan is not well understood. At least two transcription factors, the FOXOfamily transcription factor DAF-16/FOXO [7-9] and the nuclear hormone receptor DAF-12 [10], are required for germ-cell loss to extend lifespan in C. elegans [1]. When germ cells are removed, DAF-16/FOXO accumulates in nuclei, primarily in the animal's intestine [11]. The intestine, which functions as the worm's entire endoderm, including its fat tissue, appears to play a key role in this pathway, as expressing DAF-16/FOXO only in the intestine completely restores the lifespan extension of germline-defective daf-16(-) animals [12]. DAF-16/FOXO nuclear localization requires an ankyrin-repeat, intestinal protein called KRI-1 [13]. In addition, full DAF-16/FOXO nuclear localization requires
DAF-12/NHR [10,13], as well as DAF-9, a cytochrome P450 protein that synthesizes a DAF-12/NHR ligand [13-15].

DAF-16/FOXO is particularly interesting because of its evolutionarily-conserved role in another lifespan regulatory pathway, the insulin/IGF-1 endocrine pathway [16-18]. In wild-type worms, the insulin/IGF-1 receptor DAF-2 activates a series of conserved kinases that ultimately phosphorylate and inactivate DAF-16/FOXO [19-21]. When insulin/IGF-1 signaling is inhibited, DAF-16/FOXO accumulates in nuclei, where it regulates the transcription of downstream antioxidant, chaperone, innateimmunity, and metabolic genes that more directly affect lifespan [8,9,11,22-25]. Insulin/IGF-1 signaling and FOXO proteins influence lifespan in worms, flies and mice $[16,26,27]$. The pathway appears to affect human longevity as well, as variants in the human Foxo3a gene have been linked to longevity in several human populations [28-32], and functionally significant IGF-1 receptor mutations are overrepresented in populations of centenarians [33].

In an effort to better understand how reproductive cues trigger lifespan extension, we carried out a genetic screen for RNAi clones that prevent germline loss from extending lifespan. Surprisingly, in our screen we identified a gene, tcer-1, that encodes the homolog of the human transcription elongation factor TCERG1. Loss of tcer-1 in C. elegans sharply curtails the lifespan extension produced by removal of the germline, but has little or no effect on wild-type lifespan. Thus, TCER-1 is not likely to be a general component of the transcriptional machinery; instead, it has a much more specific function in the animal. 


\section{Author Summary}

The reproductive status and longevity of animals are strongly interlinked. Increasing age influences the reproductive capacities of most animals. However, little is known about how reproductive status might affect lifespan. Experiments in worms and flies have shown that removing cells that give rise to gametes, the "germ cells", makes them live longer. We know very little about the genes and molecules that are involved in this process. In this study, we have identified a gene called tcer-1 that promotes the longevity of the roundworm Caenorhabditis elegans when its germ cells are removed. The gene tcer-1 codes for a protein, TCER-1, that is predicted to function as a "transcription elongation factor" (it allows the completion of RNA synthesis during the process of gene expression). Our experiments imply that when the germ cells of worms are removed, TCER-1 collaborates with a transcription factor called DAF-16/FOXO to express genes that contribute to increased longevity. DAF-16/FOXO can extend lifespan in response to other physiological cues besides loss of germ cells. However, TCER-1 specifically helps this widely used longevity protein to respond to signals that reflect the reproductive status. Counterparts of DAF-16/FOXO are known to control aging in other organisms, including humans, so the identification of TCER-1 may lead to a better understanding of the relationship between reproduction and aging in other species, too.

We find that TCER-1 plays a key regulatory role in transducing signals from the reproductive system to the somatic tissues. When the germline is removed, TCER-1 levels rise in the intestine. This up-regulation is functionally significant because overexpressing tcer-1 in normal, fertile animals bypasses the requirement for germcell loss and extends lifespan. Our findings indicate that the role of tcer-1 in the reproductive longevity pathway is to promote the transcription of a subset of DAF-16/FOXO-target genes that are up-regulated upon germline removal. Thus, TCER-1 appears to act in association with DAF-16/FOXO to extend lifespan.

Interestingly, tcer-1 is not invariably required for DAF-16/ FOXO transcriptional activity, as it is not needed for DAF-16/ FOXO to up-regulate its target genes or to extend lifespan in daf-2 insulin/IGF-1 receptor mutants. At least some of the genes that are up-regulated by DAF-16/FOXO in a tcer-1-dependent fashion in long-lived, germline-defective animals do not require tcer-1 for their expression in long-lived insulin/IGF-1 mutants. Thus, this transcription elongation factor appears to link germline loss to a precise DAF-16/FOXO-dependent transcriptional program.

\section{Results}

\section{tcer-1 is required for the longevity associated with germline ablation}

We identified tcer-1 in a Chromosome II RNAi screen for genes required to extend the lifespan of germline-depleted $g l p-1$ mutants (see Materials and Methods). When grown at the non-permissive temperature $\left(25^{\circ} \mathrm{C}\right)$, temperature-sensitive $g l p-1$ mutants are sterile because the germline-stem cells fail to proliferate [34]. These mutants recapitulate the lifespan extension of animals whose germline-precursor cells have been eliminated by laser ablation [2]. Since lifespan can be shortened by conditions that simply compromise the animals' health, we focused on RNAi clones that suppressed the extended lifespan of $g l p-1$ mutants but had little or no effect on the lifespan of wild-type animals. From this screen, we identified a clone of the gene tcer- 1 ( $Z K 1127.9$ ), which encodes the C. elegans homolog of a human transcription elongation factor, TCERG1 (also known as CA150) [35-37]. tcer-1 RNAi strongly suppressed the extended lifespan of $g l p-1$ mutants, but had no effect on the lifespan of wild-type animals (Figure 1A and 1B; Table S1A). Wild-type animals grown on bacteria expressing tcer-1 dsRNA appeared normal and healthy, had regular developmental rates and displayed normal reproduction.

We obtained a tcer-1 mutant, tcer-1(tm1452), from the C. elegans National Bioresource Project, Japan. The tm1452 mutation is a $10 \mathrm{bp}$ insertion coupled to a $392 \mathrm{bp}$ deletion that eliminates parts of the WW domains of the protein and is likely to reduce gene function. We constructed a tcer-1; glp-1 double mutant and found that it had a much shorter lifespan than did $g l p-1$ mutants; for example, in one experiment the $74 \%$ extension in lifespan produced by the $g l p-1$ mutation was reduced by tcer-1(tm 1452) to $15 \%$ (Figure 1C, Table S1B). There was no effect on the lifespan of wild-type worms (Figure 1C, Table S1C). This finding is significant, as it indicates that tcer-1 is unlikely to play a general role in transcription elongation, but instead has a more specific function in the animal. tcer-1 mutants displayed a delay in developmental timing, and a modest reduction in brood size that was not elicited by tcer-1 RNAi treatment (data not shown).

\section{Germline loss elevates TCER-1 levels in the intestine}

The observation that reducing tcer-1 activity had only mild effects on wild type but almost completely prevented germline loss from extending lifespan suggested that tcer-1 might play a regulatory role in this pathway. If so, it seemed possible that its level or location in the animal might change upon germline removal. To investigate this possibility, we constructed transgenic animals expressing a TCER-1::GFP fusion protein under the control of the endogenous tcer- 1 promoter (see Materials and Methods). In the wild type, TCER-1::GFP was visible at all stages of embryonic and larval development (data not shown). In adults, we observed strong nuclear localization of TCER-1::GFP in intestinal cells, many head and body neurons, muscle and hypodermal cells (Figure 2A-2C). In some intestinal cells, weak expression was also observed in the cytoplasm.

To test if this fusion protein was functional, we examined the ability of the construct to rescue the shortened lifespan of tcer-1; $g l p-1$ double mutants. Two independent lines expressing the TCER-1::GFP fusion protein completely rescued the lifespan of tcer-1; glp-1 mutants in one trial (Figure 2D, Table S1C). In a second trial, the rescue was between $77-88 \%$ (Table S1C). Thus, TCER-1::GFP is a functional protein that likely reflects the endogenous expression of tcer-1. Importantly, these observations showed that a functional TCER-1 protein was present in intestinal nuclei in the adult, where DAF-16/FOXO has been shown to act to increase lifespan $[11,12]$.

Next, we examined the effect of germline ablation on the pattern of TCER-1::GFP expression. We found that eliminating the germline increased the level of TCER-1 in somatic cells (Figure 3A-3C). This finding raised the possibility that TCER-1 might have an important regulatory role in the animal's response to germline ablation. An independent microarray analysis designed to identify longevity genes whose expression is altered by germline ablation also found tcer-1 amongst the top $10 \%$ of germlineregulated genes (M. McCormick and C. Kenyon, unpublished data). Thus, tcer- 1 is likely to be transcriptionally up-regulated following germline depletion. TCER-1 levels increased primarily in two tissues, the intestine and neurons, upon germline removal (Figure 3A and 3B). tcer-1 RNAi suppressed longevity without affecting neuronal expression (Figure S1). Because neurons are 

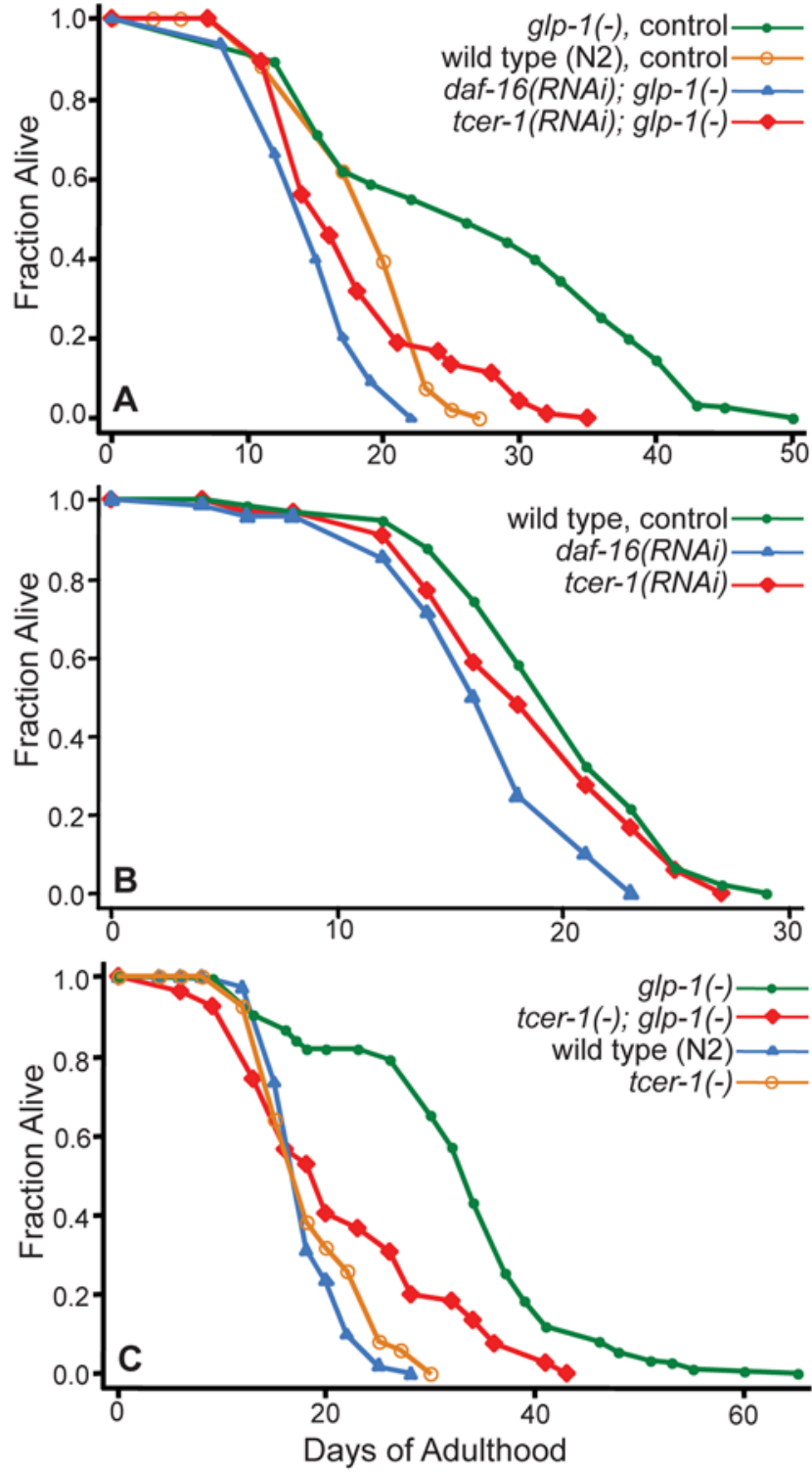

Figure 1. Reduction of tcer-1 function suppresses the lifespan extension produced by germline ablation. (A) Effect of tcer-1 RNAi on the lifespan of germline-defective mutants. glp-1 (germlinedefective) mutants grown on bacteria containing control empty vector $(m=27.0 \pm 1.1, n=112 / 114)$, daf-16 RNAi bacteria $(m=15.2 \pm 0.2$, $\mathrm{n}=110 / 113, P$ vs. control $<0.0001)$, and tcer-1 RNAi bacteria ( $m=18.1 \pm 0.4, n=96 / 114, P$ vs. control $<0.0001$ ). Wild-type (N2) worms grown on bacteria containing control empty vector shown for comparison ( $m=19.5 \pm 0.3, n=81 / 99, P$ vs. $g / p-1(-)<0.0001)$. (B) Effect of tcer-1 RNAi on the lifespan of wild-type (N2) worms. N2 worms grown on bacteria containing control empty vector $(m=19.9 \pm 0.6, n=49 / 72)$, daf-16 RNAi bacteria $(m=16.7 \pm 0.5, n=62 / 72, P$ vs. control $<0.0001)$, and tcer- 1 RNAi bacteria $(m=18.7 \pm 0.5, \mathrm{n}=66 / 72, P$ vs. control $=0.2, P$ vs. daf-16 RNAi = 0.002). (C) Effect of the tcer-1(tm 1452) mutation on the lifespan of $g / p-1$ mutants and wild-type worms. $g / p-1(-): m=32.9 \pm 0.3$, $\mathrm{n}=233 / 258$; tcer- $1(-)$; glp-1(-): $\mathrm{m}=21.8 \pm 0.5, \mathrm{n}=77 / 115, P$ vs. glp$1(-)<0.0001$; wild type (N2): $m=18.9 \pm 0.5, n=60 / 121$; tcer- $1(-)$ : $\mathrm{m}=19.0 \pm 0.4, \mathrm{n}=96 / 110, P$ vs. $\mathrm{N} 2=0.84$. Additional repeats of these experiments are shown in Table S1.

doi:10.1371/journal.pgen.1000639.g001

relatively resistant to RNAi in C. elegans, this finding suggests that intestinal TCER-1 function, like intestinal DAF-16/FOXO, is likely to be particularly important for promoting longevity. tcer-1 overexpression extends the lifespan of wild-type, fertile worms

The finding that loss of the germline elevated TCER-1 levels raised the possibility that TCER-1 might play a rate-limiting step in this longevity pathway. To test this, we asked whether overexpressing tcer-1 in wild-type animals might bypass the requirement for germline loss and extend the lifespan of fertile animals. We found that tcer-1 overexpression produced a modest but consistent increase in the lifespan of wild-type worms (average mean lifespan extension $\sim 15 \%$; Figure $3 \mathrm{D}$ and $3 \mathrm{E}$; Table S2A and S2B). We also found that, in keeping with its role in the reproductive pathway, tcer-1 overexpression did not produce any further increase in the extended lifespans of worms whose germ cells had been eliminated either by the $g l p-1$ mutation (Figure $3 \mathrm{~F}$; Table S2C) or by laser ablation (Figure S2; Table S2C).

tcer-1 is required for expression of DAF-16/FOXO-target genes in response to germline removal

To understand why tcer- 1 was required for loss of the germ cells to extend lifespan, we asked whether tcer-1 might impact DAF-16/ FOXO function. DAF-16/FOXO undergoes nuclear accumulation primarily in intestinal cells following germline removal and we first explored the possibility that tcer-1 was required for this step. However, we found that in tcer-1(tm1452) worms lacking a germline, DAF-16/FOXO accumulated normally in intestinal nuclei (Figure S3). Curiously, we noticed a small increase in the levels of DAF-16::GFP, largely in the cytoplasm of intestinal cells, in these animals (Figure S3; see Discussion).

Next, we examined the effect of ter-1(tm1452) on DAF-16/ FOXO's transcriptional output following germline loss. Many putative (direct or indirect) DAF-16/FOXO target genes have been identified [12,22-25], but only a few, sod-3, dod-8, gpd-2 and nnt -1 , are known to be up-regulated by germline removal [3]. To increase the repertoire of such genes, we obtained available transgenic worm strains expressing GFP-tagged transcriptional reporters for additional putative DAF-16/FOXO targets [38] (see Table S3). Germline precursors of each of these strains were laser ablated, and the animals were examined as adults for changes in expression. We found several genes whose expression was sharply elevated upon germline removal, including K07B1.4, T21D12.9, F52H3.5, aat-1 and pssy-1 (see Table S3).

We then asked whether the up-regulation of these new genes, as well as the known daf-16-dependent germline-regulated genes, required tcer-1. We found that this was the case for six of the nine genes we examined. The up-regulation of $g p d-2$, dod-8, nnt-1, K07B1.4, and T21D12.9 was strongly attenuated in tcer-1; glp-1 mutants (Figure 4A-4T) and pssy-1 expression was moderately reduced (data not shown). In contrast, aat-1 expression remained unaltered in two trials and showed a small increase in expression in one (data not shown). These findings suggest that loss of TCER-1 may eliminate at least a portion of DAF-16/FOXO's transcriptional response to germline depletion. Unexpectedly, sod-3 and F52H3.5 levels were further increased upon tcer-1 inactivation (Figure 4U-4X and Figure S4, respectively; see Discussion).

\section{tcer-1 overexpression up-regulates DAF-16/FOXO-target genes}

As tcer- 1 reduction of function reduced the expression of many DAF-16/FOXO-target genes, we asked if TCER-1 overexpression would increase the expression of the same DAF-16/FOXO-target genes that were downregulated in tcer-1(-); $g l p-1(-)$ mutants. Using quantitative RT-PCR (Q-PCR), we found that several such genes (dod-8, gpd-2, nnt-1, K07B1.4 and pssy-1) showed a modest but 

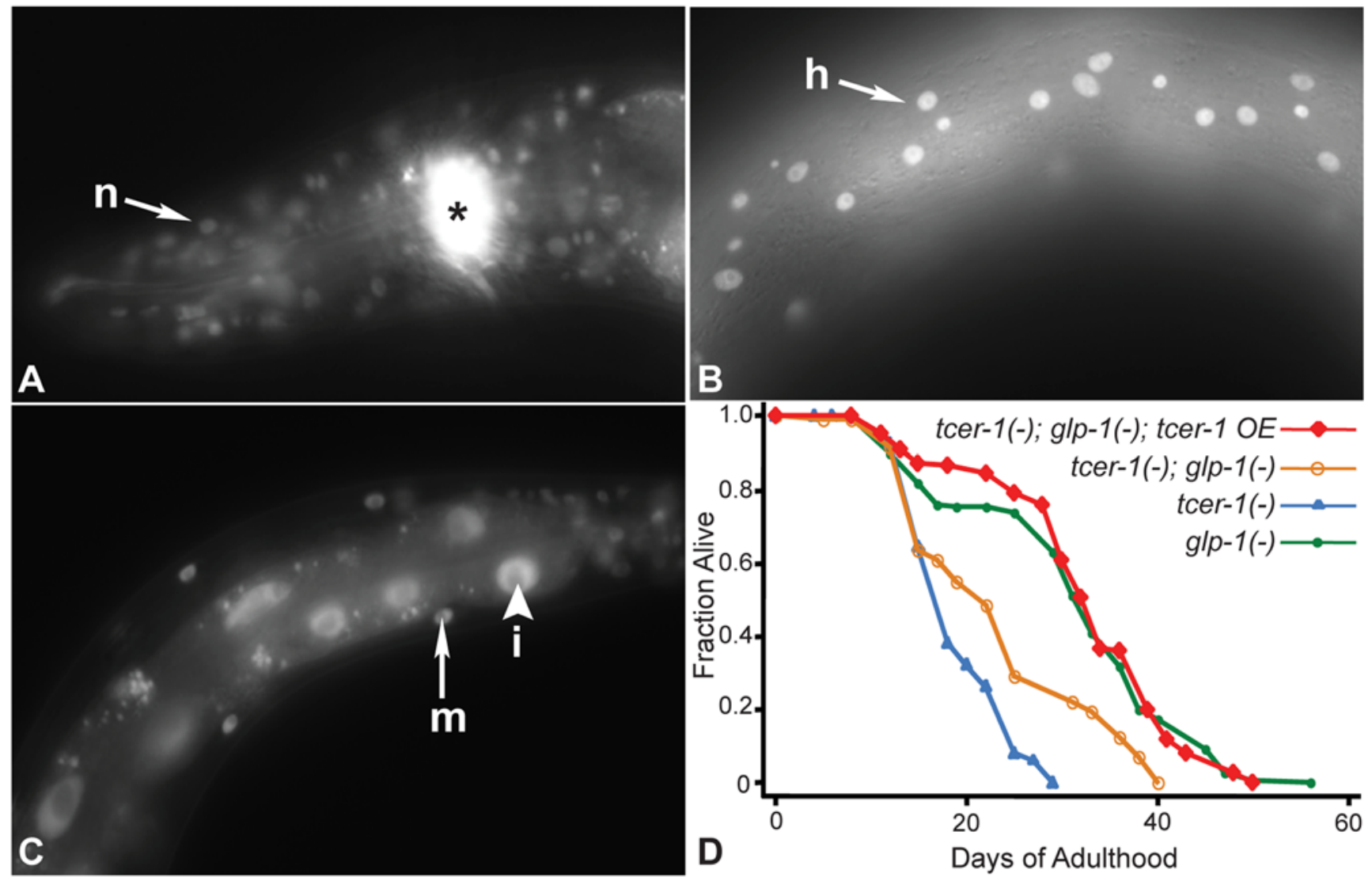

Figure 2. A TCER-1::GFP fusion protein is widely expressed and rescues the shortened lifespan of tcer-1; glp-1 mutants. (A-C) tcer-1 expression in adult somatic tissues. TCER-1::GFP is expressed in nuclei of adult neurons (' $n$ ', head neurons shown by arrow in (A)), hypodermis (' $h$ ', (B)), muscles (' $\mathrm{m}$ ', arrow in (C)), and intestinal cells (' $\mathrm{i}$ ', arrowhead in (C)). The asterisk in (A) indicates expression of the co-injection marker, Podr$1:: R F P$, in olfactory neurons. (D) Rescue of the shortened lifespan of $t c e r-1(-) ; g / p-1(-)$ mutant by the TCER-1::GFP fusion protein (tcer-1 OE). glp$1(-): \mathrm{m}=31.1 \pm 0.7, \mathrm{n}=204 / 241$; tcer-1(-); glp-1(-): $\mathrm{m}=23.4 \pm 0.5, \mathrm{n}=81 / 119, P$ vs. glp-1 $(-)<0.0001$; tcer-1 $(-)$; glp-1 $(-)$; tcer -1 OE: $\mathrm{m}=32.4 \pm 0.3$, $\mathrm{n}=111 / 116, P$ vs. $g \mid p-1(-)=0.5, P$ vs. tcer $-1(-) ; g / p-1(-)<0.0001$; tcer $-1(-): \mathrm{m}=19.1 \pm 0.2, \mathrm{n}=50 / 64$. See Table $\mathrm{S} 1$. $C$ for additional trials of this experiment.

doi:10.1371/journal.pgen.1000639.g002

statistically significant increase in expression when tcer-1 was overexpressed (Figure 5A). In addition, the lifespan extension obtained by tcer-1 overexpression required the presence of wildtype daf-16 (Figure 5B; Table S4). Together these findings suggest that tcer-1 overexpression may extend the lifespan of wild-type, fertile animals by stimulating expression of DAF16/FOXO-target genes that are induced in response to germline loss.

tcer-1 is not required for DAF-16/FOXO to extend lifespan in insulin/IGF-1 pathway mutants

Since daf-16 is also required for the doubling of lifespan produced by reducing insulin/IGF-1 signaling [16,39], we examined the role of tcer- 1 in this longevity pathway too. We found that neither tcer-1(tm1452) nor tcer-1 RNAi suppressed the extended lifespans of the insulin/IGF-1-receptor mutants daf2(e1370) or daf-2(e1368) (Figure 6A and 6B; Table S5). Therefore, TCER-1 is not part of the insulin/IGF-1 pathway. Consistent with this, we found that TCER-1 was not required in daf-2(e1370) mutants for the expression of any of the DAF-16/FOXO targets that were tcer-1-dependent in the reproductive pathway. The tcer1(tm1452) mutation either had no effect, or in some cases produced a small increase in expression (Figure 6C and 6D).

Removing the germ-cell precursors of daf-2 mutants further doubles their already long lifespan $[1,4]$. Similarly, we found that overexpressing tcer-1 in daf-2(e1370) mutants extended their long lifespan by another $6-12 \%$ (Figure $6 \mathrm{E}$; Table S2C). In contrast, we did not observe a further extension of lifespan when tcer-1 was overexpressed in germline-depleted worms (Figure 3F, Figure S2; Table S2C). These observations support the model that tcer-1 functions in the germline longevity pathway but not the insulin/ IGF-1 longevity pathway.

Not all longevity pathways in C. elegans are daf-16 dependent. For example, the longevity response to caloric restriction caused by the feeding-defective mutant eat-2(ad1116) [40] is daf-16 independent, as is the longevity response to inhibition of mitochondrial respiration caused by isp-1(qm150) [41-43]. We found that tcer-1 was not required for either of these mutations to extend lifespan (Figure $6 \mathrm{~F}$ and 6G; Table S5). These findings further reinforced the interpretation that TCER-1 is not a general longevity factor but instead extends lifespan specifically in response to reproductive signals.

The ability of TCER-1 overexpression to activate DAF-16/ FOXO-dependent gene expression and extend lifespan suggested that the up-regulation of TCER-1 is a key switch-point by which loss of the germ cells triggers a longevity response. This up-regulation could also be part of the mechanism by which TCER-1 activity is specifically directed towards this pathway and not towards the insulin/IGF-1 pathway. To test this possibility, we examined 
TCER-1::GFP
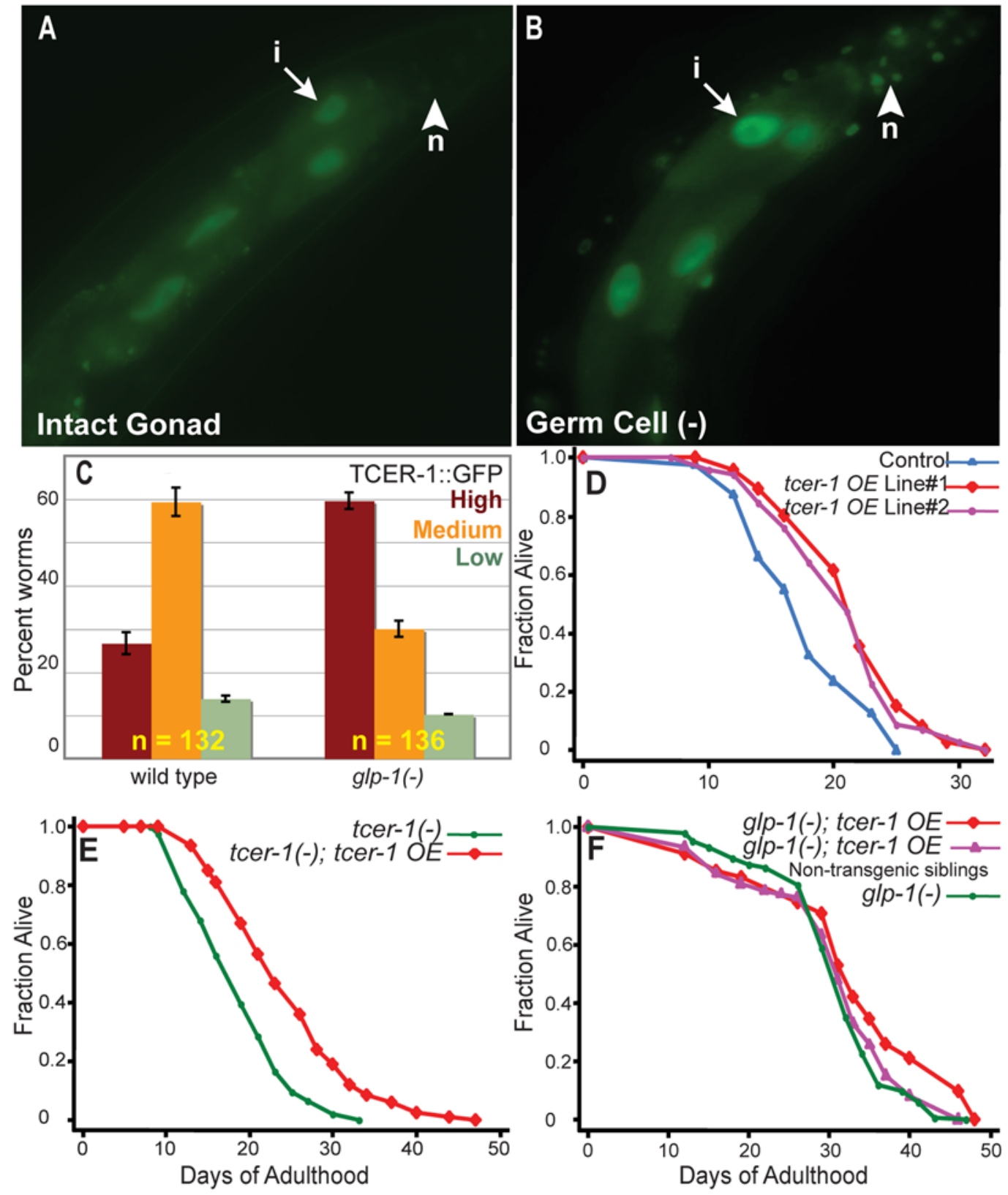

Figure 3. TCER-1 expression is elevated upon germline ablation and tcer-1 overexpression increases the lifespan of wild-type animals. (A-C) Elevation of TCER-1::GFP levels following germline removal using laser ablation (A,B) or glp-1 mutantions (C). (A,B) TCER-1::GFP expression in day 1 adults of control worms with intact gonads (A), and germ-cell precursor (Z2, Z3)-ablated worms (B). Increased expression is visible in intestinal nuclei ('i') and neurons (' $\mathrm{n}$ ') (compare arrows and arrowheads, respectively, between (A) and (B)). (C) Bar graph showing increased TCER1::GFP expression in sterile, long-lived glp-1 mutants. Day 1 adults of each strain were classified into those with High (red bars), Medium (orange bars), and Low (green bars) GFP levels (focusing primarily on the intestine). ' $n$ ' signifies the total number of worms examined in two trials. Error bars represent the standard error of the mean. (D) Effect of tcer-1 overexpression on the lifespan of intact, wild-type animals. Wild-type control carrying only the co-injection marker (Podr-1::rfp): $\mathrm{m}=17.4 \pm 0.4, \mathrm{n}=81 / 89$. TCER-1::GFP, tcer-1 OverExpressing strains (tcer-1 OE), Line \#1: $\mathrm{m}=21.2 \pm 0.4, \mathrm{n}=85 /$ 90, $P$ vs. control $<0.0001$; Line $\# 2: m=21.6 \pm 0.6, n=74 / 79, P$ vs. control 0.0002 . (E) Effect of tcer-1 overexpression on the lifespan of tcer-1 mutants. tcer-1(-): $\mathrm{m}=18.6 \pm 0.4, \mathrm{n}=91 / 108$; tcer-1(-); tcer-1 OE: $\mathrm{m}=24.6 \pm 0.8, \mathrm{n}=81 / 106, P<0.0001$. tcer-1 overexpression in a tcer-1(-) background produced a greater lifespan extension than tcer-1 overexpression in tcer-1(+) background. For example, in the above experiment, tcer-1 overexpression in a tcer-1 (-) background produced a 33\% lifespan extension as compared to $14 \%$ in a tcer-1(+) background (note the difference in Xaxis scale between $\mathrm{A}$ and $\mathrm{B}$ ). The reason for this difference is unclear, but it is similar to the trend exhibited by another longevity-promoting transcription factor, SKN-1 [68]. (F) Effect of tcer-1 overexpression on the lifespan of $g / p-1$ mutants. $g / p-1(-): 30.6 \pm 0.2, \mathrm{n}=102 / 104$. glp-1(-); tcer-1 OE: $\mathrm{m}=30.4 \pm 0.3, \mathrm{n}=82 / 89, P$ vs. $g / p-1(-)$ 0.3. $g / p-1(-)$; tcer-1 OE non-transgenic (wild-type) siblings: $\mathrm{m}=30.6 \pm 0.2, \mathrm{n}=86 / 89, P$ vs. $g / p-1(-) 0.4, P$ vs. $g / p-$ $1(-)$; tcer-1 $O E=0.2$. See Table S2 for results with additional transgenic lines and multiple repeats of above experiments. doi:10.1371/journal.pgen.1000639.g003 


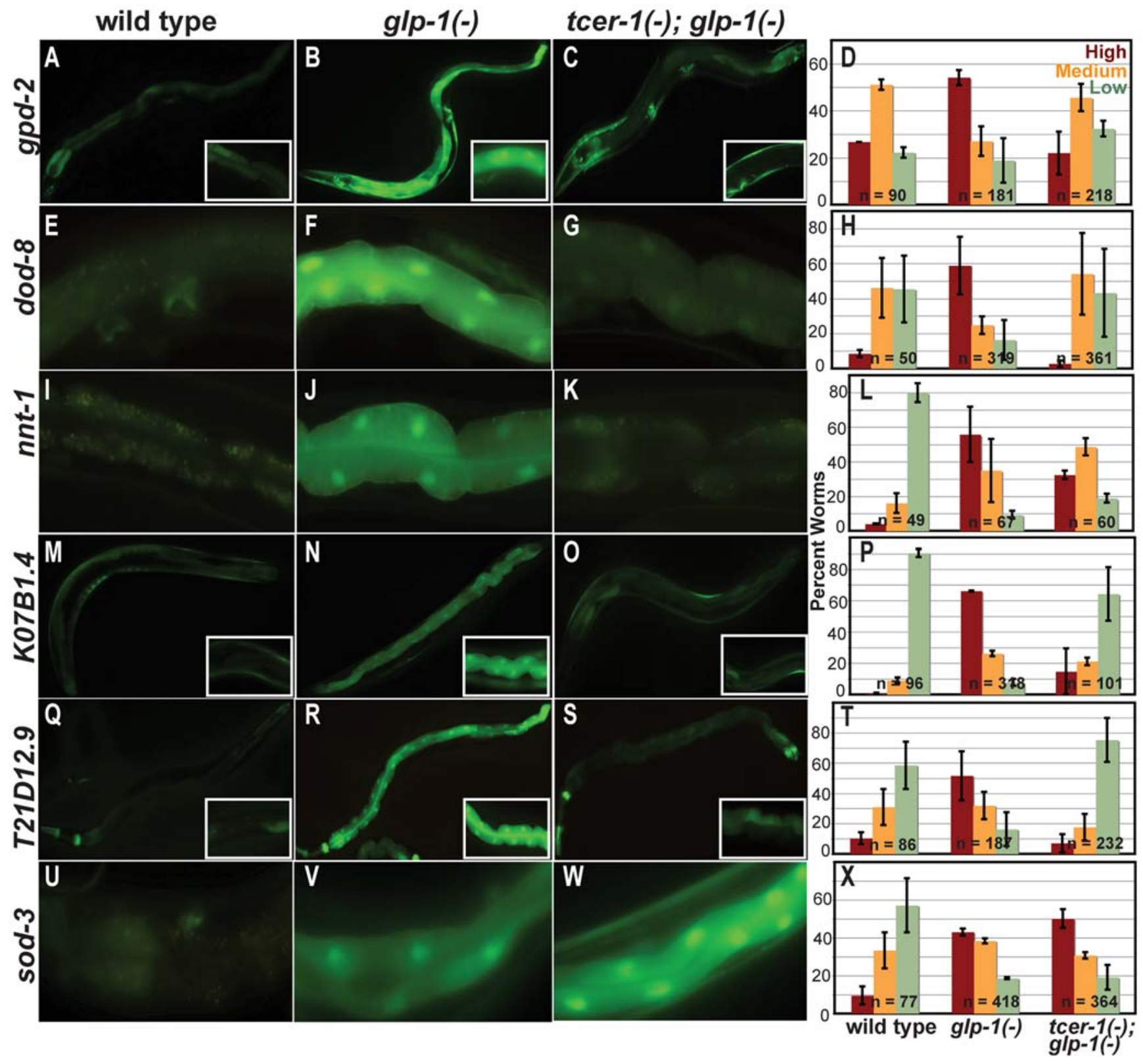

Figure 4. $t c e r-1$ is required for the up-regulation of DAF-16/FOXO-target genes in g/p-1 mutants. (A-X) Expression of DAF-16/FOXOtarget genes in wild-type worms (left column), glp-1 mutants (middle column), and tcer-1; glp-1 mutants (right column). Animals were observed on day 2 of adulthood using transcriptional GFP reporters for individual genes. Bar graph representation of the data for each gene is shown in the farright column. In each experiment, worms were classified into those with High (red bars), Medium (orange bars), and Low (green bars) GFP levels. ' $n$ ' signifies the total number of worms examined in two or three trials. Error bars represent the standard error of the mean. The elevated expression observed in glp-1 mutants was reduced in the case of gpd-2 (A-D), dod-8 (E-H), nnt-1 (I-L), K07B1.4 (M-P) and T21D12.9 (Q-T). Expression of sod-3 was elevated further $(U-X)$. In $(A-C),(M-O)$, and $(Q-S)$, images were observed at $200 \times$ magnification. The insets in these panels show intestinal cells of the same strains imaged at $400 \times . \ln (E-G),(I-K)$, and $(U-W)$, images of intestinal cells were observed at $400 \times$. For more information about the expression patterns of these genes, and other DAF-16/FOXO-targets, see Table S3. doi:10.1371/journal.pgen.1000639.g004

TCER-1::GFP levels in daf-2(e1370) mutants. We found that daf-2 mutants did not have elevated TCER-1::GFP levels. In contrast, TCER-1 levels were significantly reduced (Figure 6H, compare with Figure 3C). TCER-1 levels were also reduced in long-lived eat2(ad1116) mutants (Figure S5), and we found that tcer-1 overexpression further extended the lifespan of eat-2(ad1116) mutants (Figure S5; Table S2C). These data suggested that the specificity of TCER-1 for the reproductive pathway might be achieved, at least in part, by the regulation of its level in the animal. kri- 1 controls the intestinal expression of tcer- 1

How is TCER-1 up-regulation controlled by the reproductive system? To begin to address this question, we asked whether daf-16 itself, or genes that are known to influence DAF-16/FOXO nuclear localization in response to germline loss [13], were involved. We found that the daf-16(mи86) null mutation did not alter TCER-1::GFP levels in wild-type or in germline-deficient animals (Figure 7A). Likewise, neither daf-9 nor daf-12 mutations, both of which partially inhibit DAF-16/FOXO nuclear localiza- 

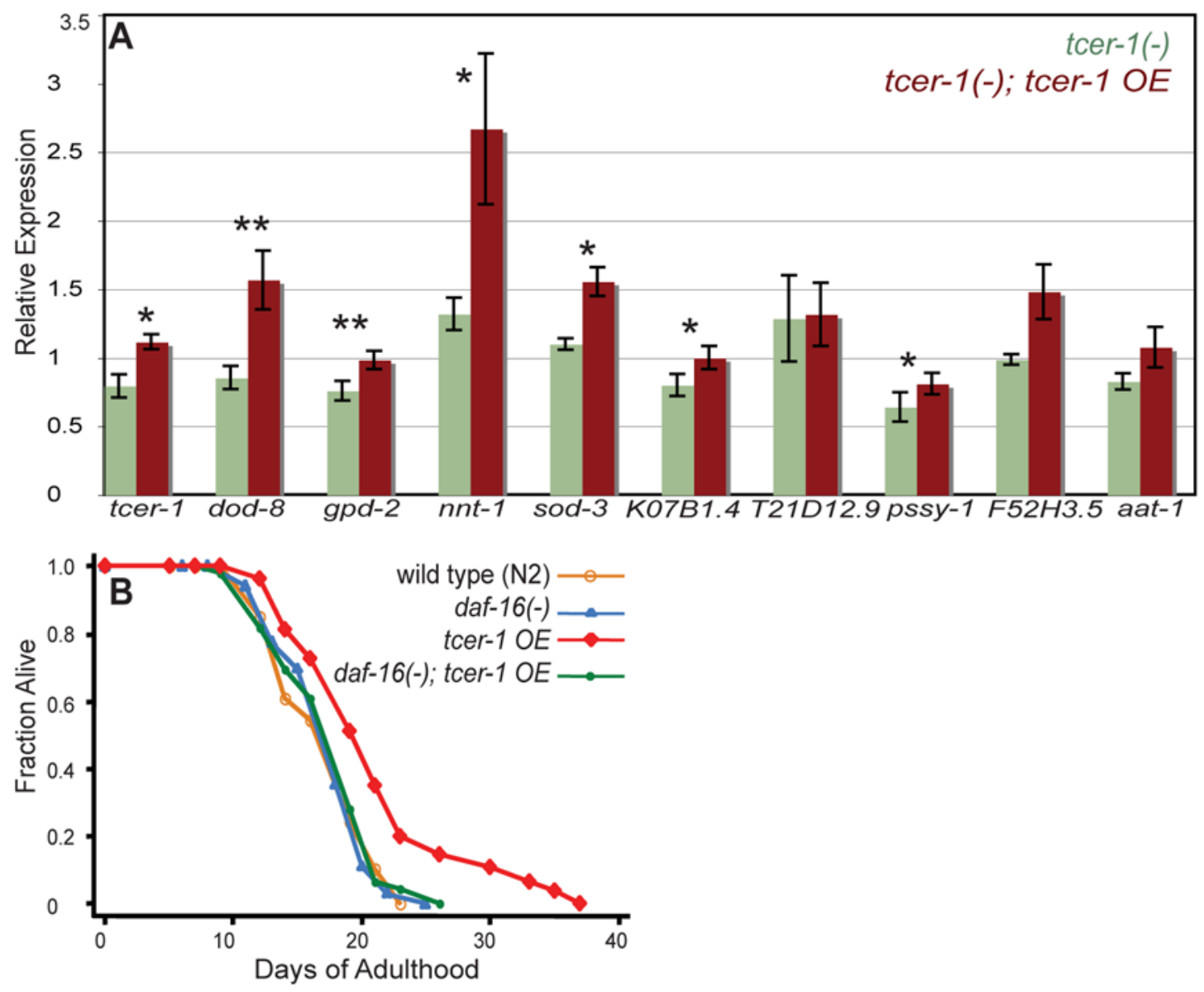

Figure 5. tcer-1 overexpression causes increased transcription of DAF-16/FOXO-target genes. (A) mRNA levels of DAF-16/FOXO-targets compared between tcer-1(-) (green bars) and tcer-1(-); tcer-1 OE (red bars) animals. Day 1 adults grown under similar conditions were analyzed by Q-PCR. Data shown here is combined from six independent biological replicates, each comprising 2-4 technical repeats. The genes tested are displayed on the $\mathrm{X}$-axis and relative expression levels are on the $\mathrm{Y}$-axis. Error bars display standard error of the mean. The asterisks represent a change in expression between the two strains with an unpaired, two-tailed $t$-test with a $P$ value $<0.05\left(^{*}\right)$ or $<0.005\left(^{* *}\right)$. (B) Lifespan extension mediated by tcer-1 overexpression depends on daf-16. Wild type (N2): $\mathrm{m}=18.4 \pm 0.3, \mathrm{n}=66 / 98 ;$ daf- 16 (mu86): $\mathrm{m}=17.5 \pm 0.2, \mathrm{n}=65 / 81, P$ vs. N2 $=0.03 ;$ tcer-1 OE: $\mathrm{m}=21.1 \pm 0.2, \mathrm{n}=79 / 102, P$ vs. $\mathrm{N} 2<0.0001$; daf-16(mu86); tcer $-1 \mathrm{OE}: \mathrm{m}=17.6 \pm 0.2, \mathrm{n}=89 / 104, P$ vs. $\mathrm{N} 2=0.54, P$ vs. daf- 16 (mu86) $=0.15, P$ vs. tcer- 1 $O E<0.0001$. See Table $S 4$ for multiple repeats of this experiment. doi:10.1371/journal.pgen.1000639.g005

tion in germline-defective animals, affected TCER-1 up-regulation (data not shown). However, in kri-1(ok1251) mutants, nuclear TGER-1::GFP levels were severely diminished in the intestine and were not increased following germ-cell ablation (Figure 7B-7F). $k r i-1(o k 1251)$ did not alter TCER-1::GFP levels in any other tissues (Figure 7E and 7F), consistent with KRI-1's presence only in the gastrointestinal tract [13]. Thus, DAF-16/FOXO nuclear localization and TCER-1 up-regulation share a requirement for KRI-1 in their response to germ-cell loss. As expected, kri-1(ok1251) also abolished the lifespan extension evoked by tcer-1 overexpression (Figure 7G; Table S4). KRI-1 is not required for DAF-16/FOXO nuclear localization or lifespan extension in daf-2 mutants [13]. Thus, KRI-1's involvement in TCER-1 regulation explains, at least in part, why TCER-1 function is specific for the reproductive system.

\section{Discussion}

The finding that signals from reproductive tissues can influence the lifespan of an animal is fascinating and important because both reproduction and aging are such fundamental aspects of an animal's life history. In this study, we have shown that a predicted transcription elongation factor, TCER-1, is a key regulatory target of lifespan-extending signals from the reproductive system in C. elegans. TCER-1's level in the animal is sensitive to cues from the reproductive system, and when overexpressed, TCER-1 can bypass the normal requirement for germline loss and extend the lifespan of fertile animals with an intact reproductive system.

Our data indicate that TCER-1 extends lifespan by collaborating with the DAF-16/FOXO transcription factor, which is also required for the loss of germ cells to extend lifespan [1]. DAF-16/ FOXO has an evolutionarily-conserved role in lifespan regulation $[18,44]$, and is activated in many other circumstances that increase lifespan, including reduced insulin/IGF-1 signaling [8,11] or overexpression of the sirtuin SIR-2.1 [45], heat-shock factor HSF1 [46], AMP kinase [47] or c-Jun N-terminal kinase [48]. How such a core, conserved longevity factor is activated by many disparate stimuli is a central question in the biology of aging. In this study, we have shown that TCER-1 plays a key role in establishing a distinct pattern of DAF-16/FOXO-dependent gene expression specifically in response to signals from the germline. 

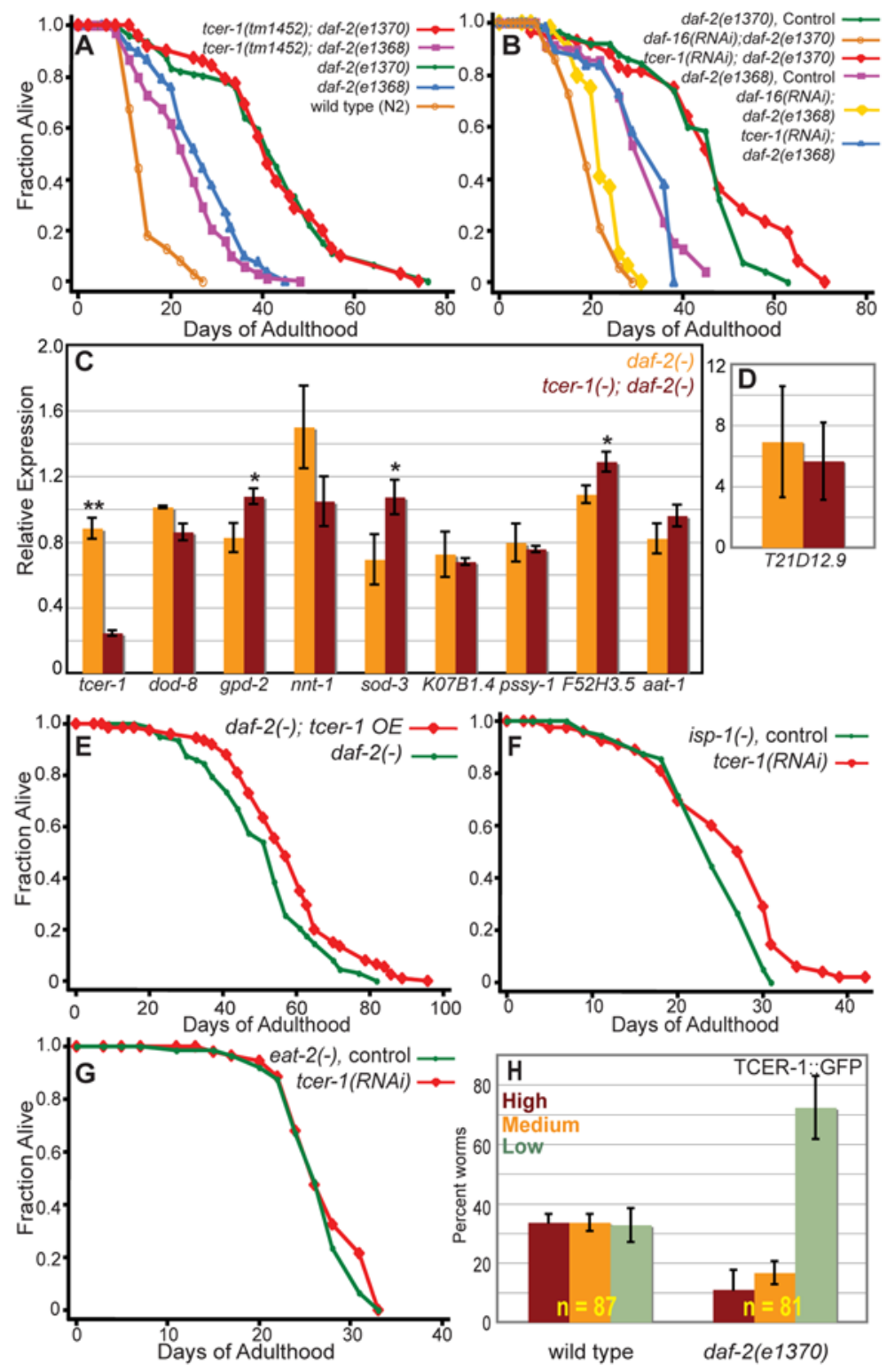

Figure 6. tcer-1 reduction of function does not suppress the extended lifespans of other longevity mutants. (A) Effect of $t c e r-1(t m 1452)$ on longevity of daf-2(e1370) and daf-2(e1368) mutants. Lifespan of daf-2(e1370): $\mathrm{m}=47.2 \pm 0.7, \mathrm{n}=91 / 103$; tcer-1(tm1452); daf-2(e1370): $\mathrm{m}=46.6 \pm 0.6$, $\mathrm{n}=58 / 102, P=0.43$. daf-2(e1368): $\mathrm{m}=33.1 \pm 0.4, \mathrm{n}=75 / 103$; tcer-1(tm1452); daf-2(e1368): $\mathrm{m}=31.3 \pm 1.8, \mathrm{n}=57 / 100, P=0.1 . \mathrm{N} 2: \mathrm{m}=16.7 \pm 0.1, \mathrm{n}=70$ / 113. (B) Effect of tcer-1 RNAi on longevity of daf-2(e1370) and daf-2(e1368) mutants. Lifespan of daf-2(e1370) mutants grown on bacteria containing empty control vector $(m=45.6 \pm 1.5, n=58 / 77)$, daf-16 RNAi bacteria $(m=16.2 \pm 0.1, n=45 / 73, P$ vs. control $<0.0001)$, and tcer -1 RNAi bacteria $(m=47.5 \pm 2.0, n=45 / 63, P$ vs. control $=0.12)$. daf-2(e1368) mutants grown on bacteria containing empty control vector $(m=31.0 \pm 1.3, n=47 / 76)$, daf16 RNAi bacteria $(m=19.7 \pm 0.4, n=35 / 69, P$ vs. control $<0.0001)$ and tcer-1 RNAi bacteria $(m=32.0 \pm 0.8, n=41 / 75, P$ vs. control $=0.54)$. $(C, D)$ tcer1 (tm 1452) does not suppress the up-regulation of DAF-16/FOXO-target-genes in daf-2(e1370) mutants. mRNA levels of DAF-16/FOXO-regulated genes were compared between daf-2(e1370) mutants (orange bars) and tcer-1(tm1452); daf-2(e1370) mutants (red bars). Day 1 adults grown under similar conditions were tested by Q-PCR analysis. The data shown here are combined from three independent biological replicates, each comprising $2-4$ technical repeats. Genes tested are displayed on the $\mathrm{X}$-axis and relative expression levels are on the $\mathrm{Y}$-axis. Error bars display standard error of the mean. The asterisks represent a change in expression between the two strains that have a $P$ value $<0.01\left(^{*}\right)$ or $<0.005(* *)$ using an unpaired, twotailed $t$-test. The expression of T21D12.9 has been depicted in a separate panel (D) in the interest of clarity, as this gene is expressed at higher levels than the others. (E) Effect of tcer-1 overexpression on the lifespan of daf-2(e1370) mutants. daf-2(-): $\mathrm{m}=51.2 \pm 1.5, \mathrm{n}=63 / 87$; daf-2(-); tcer-1 OE: $\mathrm{m}=57.6 \pm 1.4, \mathrm{n}=74 / 90, P=0.01$. See Table S2 for additional trials of this experiment. (F) Effect of tcer-1 RNAi on longevity of isp-1(qm150) mutants. Lifespan of isp-1(qm150) mutants grown on bacteria containing empty control vector $(\mathrm{m}=24.1 \pm 0.5, \mathrm{n}=39 / 91)$ and tcer-1 RNAi bacteria $(\mathrm{m}=26.1 \pm 0.5, \mathrm{n}=52 / 90, P$ vs. control $=0.0005)$. See Table $\mathrm{S} 5$ for multiple repeats of the lifespan experiments shown above. (G) Effect of tcer-1 RNAi on longevity of eat-2(ad1116) mutants. Lifespan of eat-2(ad1116) mutants grown on bacteria containing empty control vector ( $m=26.5 \pm 0.4, \mathrm{n}=59$ / 72) and tcer-1 RNAi bacteria ( $m=27.2 \pm 0.5, n=40 / 71, P$ vs. control 0.2). (H) Expression of tcer-1 in daf-2(e1370) mutants. Bar graph showing reduced TCER-1::GFP expression in long-lived daf-2(e1370) mutants. Day 1 adults of each strain were classified into those with High (red bars), Medium (orange bars), and Low (green bars) GFP levels (focusing primarily on the intestine). ' $n$ ' signifies the total number of animals examined in two trials. Error bars represent the standard error of the mean.

doi:10.1371/journal.pgen.1000639.g006 


\section{TCER-1::GFP}
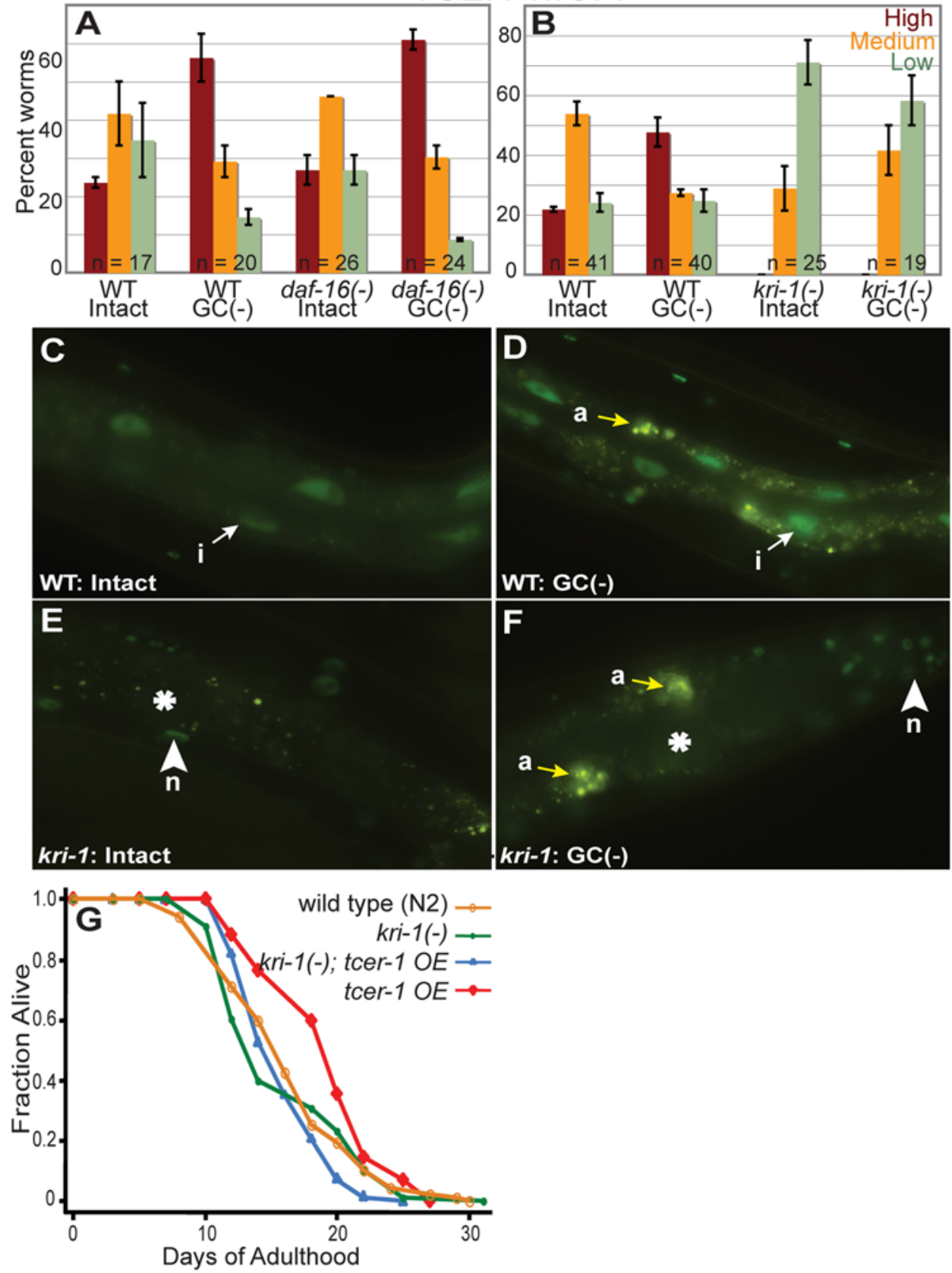

Figure 7. TCER-1 expression is elevated upon germline ablation in a daf-16-independent and $k r$ i- 1 -dependent fashion. (A) TCER-1 upregulation does not require daf-16. Bar graph showing increased TCER-1::GFP levels in animals with germline-precursor cells (Z2, Z3)-ablated in a wildtype background (WT) or in daf-16(mu86) mutants. Day 1 adults of each strain were classified into those with High (red bars), Medium (orange bars), and Low (green bars) GFP levels (focusing primarily on the intestine). ' $n$ ' signifies the total number of animals examined in two trials. Error bars represent the standard error of the mean. (B-F) Intestinal TCER-1 levels are influenced by kri-1. TCER-1::GFP expression observed in day 1 adults of wild-type control (WT) worms (C,D) with intact gonads (C), and following germ-cell precursor (Z2, Z3)-ablation (D). Increased expression in intestinal nuclei (i) is visible (compare white arrows between (C) and (D)). Images of age-matched intact (E) or germline-ablated (F) kri-1(ok1251) mutants are shown. In both cases, there is drastic reduction in intestinal GFP (asterisks). In some trials, we observed a small increase in the cytoplasmic GFP levels in intestinal cells following germline ablation, but this was not quantifiable due to the strong reduction in overall GFP signal in the intestine. Autofluorescence (a) in intestinal cells is indicated by yellow arrows. kri-1(ok1251) did not alter TCER-1::GFP in any other tissues such as neurons ( $\mathrm{n}$, arrowheads). Bar graph of data shown in (C-F) is depicted in (B) (key and experimental set up same as in (A)). (G) Lifespan extension produced by tcer1 overexpression requires kri-1. N2: $\mathrm{m}=16.5 \pm 0.3, \mathrm{n}=90 / 138 ; k r i-1$ (ok1251): $\mathrm{m}=15.9 \pm 0.2, \mathrm{n}=69 / 135, P$ vs. N2 $=0.19 ;$ tcer-1 OE: $\mathrm{m}=20.5 \pm 0.4, \mathrm{n}=97$ / $130, P$ vs. $\mathrm{N} 2=<0.0001, P$ vs. kri-1(ok1251) $<0.0001$; kri-1(ok1251); tcer- 1 OE: $\mathrm{m}=16.0 \pm 0.5, \mathrm{n}=79 / 128, P$ vs. $\mathrm{N} 2=0.54, P$ vs. $k r i-1$ (ok1251) $=0.46, P$ vs. tcer-1 OE $<0.0001$. See Table S4 for multiple repeats of this experiment. doi:10.1371/journal.pgen.1000639.g007 


\section{Model for TCER-1 function}

We can suggest hypotheses about the molecular function of TCER-1 from studies of its human homolog, the transcription elongation factor TCERG1 (also known as CA150) [35,36]. TCERG1 is known to associate with elongation-competent RNA Polymerase II (RNAPII) complexes and alter the elongation efficiency of nascent mRNA transcripts $[36,49,50]$. It has also been implicated in splicing [51-53]. TCERG1 (like TCER-1) has a modular structure, with FF repeats that associate with the $\mathrm{C}$ Terminal Domain (CTD) of RNAPII, and WW domains that can be used for association with other regulatory proteins $[37,50,51,53]$, consistent with its role in regulating gene expression.

The known function of TCERG1 suggests the model that TCER-1 influences longevity by promoting transcript elongation of DAF-16/FOXO-target genes. Specifically, we propose that following germline ablation, DAF-16/FOXO accumulates in nuclei and initiates transcription of a set of downstream targets, many of which require TCER-1 for transcript elongation. In germline-ablated tcer-1 mutants, the transcription of such genes is stalled, so lifespan extension is prevented. This hypothesis is supported by recent discoveries of transcription elongation factors that selectively influence transcription initiated by particular transcription initiation (activation) factors $[54,55]$. FOXO proteins are known to be regulated by many types of covalent modifications, including phosphorylation on multiple sites, deacetlyation, and in addition by regulated proteolysis [56,57]. To our knowledge, this is the first suggestion of DAF-16/FOXO regulation exerted at the level of transcription elongation. However, it is important to note that our experiments, which utilized GFP fusions and RT-PCR (that report on the efficiency of transcription in general and not the elongation step in particular), do not rule out the possibility that tcer- 1 acts at a different stage of transcription, such as transcription initiation.

Unexpectedly, we noticed that the expression of some DAF-16/ FOXO target genes, such as sod-3 and F52H3.5, was further increased in tcer-1(-); glp-1(-) mutants (Figure 4U-4X and Figure S4). We also found that the cytoplasmic levels of DAF-16::GFP were elevated in these animals (Figure S3). One explanation for these observations is that in germline-depleted tcer-1 mutants, the silence of many DAF-16/FOXO targets triggers a compensatory response that elevates DAF-16/FOXO levels in the cytoplasm (and possibly to some extent in the nucleus as well). For tcer-1dependent genes (such as nnt-1), this elevated DAF-16/FOXO cannot compensate for the absence of TCER-1, and their transcription remains stalled. However, for tcer-1-independent genes (such as sod-3), elevated DAF-16/FOXO levels increase transcription.

\section{DAF-16/FOXO activates different patterns of gene expression in response to insulin/IGF-1-pathway inhibition and germline loss}

Since DAF-16/FOXO increases lifespan in response to germline loss and reduced insulin/IGF-1 signaling, a simple model would be that it activates the same downstream lifespan-extending genes in both situations. However, we found that at least some of the genes that are up-regulated in germline-defective mutants in a tcer-1 dependent manner are not up-regulated in daf-2 mutants (for example T21D12.9; see Table S3). This finding suggests that the differential deployment of tcer-1 activity allows reproductive cues and reduced insulin/IGF-1 levels to trigger different patterns of DAF-16/FOXO-dependent gene expression. Thus, this situation illustrates how the activities of broadly-deployed transcription factors can be tailored to precise transcriptional outputs in response to specific signals. Interestingly, some genes that are up-regulated in germline-defective mutants in a tcer-1-dependent way are also up-regulated in daf-2 mutants, but independently of tcer-1 (for example, dod-o). Perhaps a different transcriptionelongation factor, one that can substitute for TCER-1, is activated by reduction of insulin/IGF-1 activity.

\section{Activation of the reproductive longevity pathway in animals that have germ cells}

A major question in the field of aging is whether a mode of lifespan extension that is normally triggered by one specific condition, such as caloric restriction, can be triggered instead by intervention at a downstream regulatory step of the pathway. As described above, we found that the longevity pathway activated by loss of the germ cells could potentially operate independently of signals related to reproduction. The finding that germline removal triggers the transcriptional up-regulation of tcer- 1 suggested that this regulatory step might function as a germline-pathway specific switch that activates downstream longevity processes. In fact this appears to be the case: when tcer-1 is overexpressed in intact animals that have germ cells, lifespan is extended. This lifespan extension has no detrimental effects on progeny production (data not shown) and is accompanied by several hallmarks of the germline-longevity pathway, including a requirement for KRI-1 and DAF-16/FOXO, and the stimulation of a pattern of gene expression similar to that produced by germline removal. The lifespan increment obtained by overexpressing tcer-1 was not as large as that produced by germ-cell loss. Nevertheless, these data, and other recent observations [58], suggest that at least some of the beneficial longevity effects produced by loss of the germline can be replicated without perturbing reproduction itself.

\section{Perspective}

The last few decades have transformed our view of aging from a haphazard, unregulated phenomenon to a process influenced by conserved genetic pathways. The insulin/IGF-1 pathway and DAF-16/FOXO orthologs have been implicated in lifespan regulation in worms, flies, mice, dogs and several human populations [16,26-33,59,60]. Studies in flies and mice indicate that pathways linking reproduction and aging maybe widespread in nature too. Given this context, the identification of a regulator, TCER-1, that selectively responds to reproductive signals to influence the transcriptional response of a conserved longevitydeterminant, DAF-16/FOXO, is exciting. It will be interesting to learn whether TCER-1's function in aging has also been conserved during evolution.

\section{Materials and Methods}

\section{Strains}

Strains were maintained as described earlier [61]. The tcer1(tm1452) mutant was provided by the National Bioresource Project (Japan) and outcrossed three times to the Kenyon Lab N2 stock. Transgenic strains expressing GFP under control of promoters of DAF-16/FOXO-target genes were obtained from the CGG, or from Prof. David Baillie's laboratory (Caenorhabditis Gene Expression Consortium). The details of these transgenic animals, strains generated using them and other strains used in this study are described in Table S6 and Table S3.

\section{Molecular biology}

The Ptcer-1::tcer-1::gfp fusion construct was generated as described previously [62]. The complete coding sequence of tcer- 
$1(4.1 \mathrm{~kb})$ and $1.6 \mathrm{~kb} 5^{\prime}$ upstream promoter sequence were amplified using the following primers: Forward- 5' GCA AGT ATT TGA GGA CTA CTG TCA AGG GC 3', Reverse- 5' AGT CGA CCT GCA GGC ATG CAA GCT TTG CTT TCT GGG ATC CGG CTC $3{ }^{\prime}$. The $g f p$ construct $(1.9 \mathrm{~kb})$ was amplified from the vector pPD95.75 using the following primers: Forward- 5' AGC TTG CAT GCG TGC AGG TGG ACT 3', Reverse- 5' AAG GGC CGG TAG GGC CGA CTA GTA GG $3^{\prime}$. The complete $g f p$ fusion construct $(7.6 \mathrm{~kb})$ was amplified by pooling the products of the above two PGRs and using the following primers: Forward: 5' GGC GGT CAT GGT CTT CTT CAA C 3', Reverse- 5' GGA AAG AGT TAT GTT TGG TAT ATT GGG AAT GTA TTG TG $3^{\prime}$. The co-injection marker Podr-1::rfp was amplified from the plasmid pCF155.

\section{Transgenic strains}

To generate the TCER-1::GFP expressing worms, Ptcer-1::tcer$1:: g f p$ was injected as described earlier [63] at $50 \mathrm{ng} / \mu \mathrm{l}$ or $20 \mathrm{ng} /$ $\mu \mathrm{l}$, along with the co-injection marker Podr-1::rfp (75 ng/ $\mu \mathrm{l})$, into N2 worms (see Table S6). As a control, Podr-1::rfp alone was injected into $\mathrm{N} 2$ worms $(75 \mathrm{ng} / \mu \mathrm{l})$. The resulting transgenic control strains (CF2144, CF2145) had mean lifespans that were the same as N2.

\section{Lifespan analysis}

Lifespan assays in general were conducted as previously described [64]. For $g l p-1$ mutant lifespan assays, eggs were incubated at $20^{\circ} \mathrm{C}$ for $2-6 \mathrm{hrs}$, transferred to $25^{\circ} \mathrm{C}$ to eliminate germ cells, then shifted back to $20^{\circ} \mathrm{C}$ on day 1 of adulthood for the rest of their lifespan. All other lifespan assays were performed at $20^{\circ} \mathrm{C}$. In all cases, the L4 stage was counted as day 0 of adulthood. In all experiments with TCER-1::GFP, worms were examined for Podr-1::rfp co-injection marker under a Leica MZ16F stereomicroscope (Wetzlar, Germany) on day 0 and isolated for lifespan experiments. The non-transgene carrying siblings were used as negative controls in the same experiment. $g l p-1$ mutant strains used in lifespan assays were completely sterile. Fertile strains were transferred every other day to fresh plates until progeny production ceased. Animals that crawled off the plate, exploded, bagged, or became contaminated were censored. Stata 10.0 and 8.2 (Stata Corporation, Texas, USA) and (for some lifespans) Statview 5.0.1 (SAS) softwares were used to calculate mean life spans and perform statistical analyses. $P$ values were determined using log-rank (Mantel-Cox) statistics.

\section{RNAi experiments}

We screened a C. elegans chromosome II RNAi library to identify RNAi clones that suppressed the extended lifespan of $g l p-1$ mutants. Details of a similar screen were described earlier [13]. All RNAi experiments were performed as described previously $[65,66]$. In general, RNAi clones were inoculated overnight at $37^{\circ} \mathrm{C}$ in $\mathrm{LB}$ medium containing $10 \mu \mathrm{g} / \mathrm{ml}$ tetracycline and $100 \mu \mathrm{g} / \mathrm{ml}$ carbenicillin, and seeded onto NG-carbenicilin plates supplemented with 0.1 M IPTG. For all lifespan experiments, and the RNAi screen, worms were exposed to RNAi clones from hatching. All RNAi clones were confirmed by sequencing (M13forward primer) and upon start of every experiment, by PCR (T7 primers). Clones were obtained from the libraries described previously $[65,66]$. pAD 12 (empty vector) was used as the negative control, and pAD43 (daf-16 RNAi) as the positive control for the screen and for individual lifespans [43]. The tcer-1 RNAi clone also targets a partial duplication of tcer-1, the gene $Z K 1127.6$, whose predicted protein product contains only FF domains. Transcriptional and full-length translational GFP-tagged reporters for
ZK1127.6 showed no basal expression or induction on germline ablation suggesting that it is a non-functional duplication of tcer-1 (ZK1127.9).

\section{Laser ablations}

Laser ablations of germ-cell precursors (Z2, Z3) were performed using a Zeiss Axiophot with a laser attachment (Photonics Instruments, USA) as described earlier [67]. Briefly, eggs were transferred to fresh plates and left at $20^{\circ} \mathrm{C}$ for $1-3 \mathrm{hrs}$. Hatched L1 larvae were mounted on slides with $2 \%$ Agarose pads containing $1.5 \mathrm{mM}$ Sodium Azide anesthetic. As intact controls, worms were subjected to all the steps of this process except for ablation. Ablated worms (and controls) were removed from the slide and grown at $20^{\circ} \mathrm{C}$ until required for GFP assays or lifespan analysis.

\section{GFP assays}

Eggs of worms carrying the Pdaf-16::daf-16::gfp reporter construct and the different DAF-16/FOXO-target gene reporters (in wild-type, $g l p-1$ mutant and tcer-1; $g l p-1$ mutant backgrounds) were incubated at $20^{\circ} \mathrm{C}$ for $2-6 \mathrm{hrs}$, transferred to $25^{\circ} \mathrm{C}$ to eliminate germ cells, then shifted back to $20^{\circ} \mathrm{C}$ on day 1 of adulthood. GFP assays were conducted on day 2 of adulthood, using a Leica MZ16F (Wetzlar, Germany) stereomicroscope with standard fluorescence filter sets. All assays were performed blind after initial familiarization of GFP levels in control plates by the experimenter.

\section{Microscopy}

All fluorescence images were captured using a Retiga EXi Fast1394 CCD digital camera (QImaging, Burnaby, BC, Canada) attached to a Zeiss Axioplan 2 compound microscope (Zeiss Corporation, Jena, Germany). Openlab 4.0.2 software (Improvision, Coventry, U.K.) was used for image acquisition. GFP assays were conducted on a Leica MZ16F (Wetzlar, Germany) stereomicroscope with fluorescence filter sets or (in the case of Pnnt-1::gfp) the Zeiss Axioplan 2 compound microscope mentioned above. Preliminary image processing was performed using Photoshop 10 (Adobe Creative Suite 3, USA).

\section{Q-PCRs}

Total RNA was isolated from synchronized populations of approximately 15,000 day 1 daf-2 and tcer- 1 ; daf-2 mutants. For comparing, tcer-1(-) and tcer-1(-); tcer-1 OE strains, worms were picked manually for RNA isolation. Eggs were transferred to fresh plates. Day 0 (L4) transgenic animals carrying tcer-1(-); tcer-1 OE were isolated using a Leica MZ16F stereomicroscope with standard fluorescence filters. 2,000-5,000 worms were picked manually for RNA isolation for each biological replicate. A similar number of tcer1 mutants were also collected manually. Both strains were allowed to grow for $24 \mathrm{hrs}$ and on day 1 of adulthood used for RNA isolation. Total RNA was extracted using TRIzol reagent (Invitrogen) and purified using Qiagen RNAeasy Mini Kit. cDNA was generated using Protoscript First Strand cDNA Synthesis Kit (New England Biolabs). SybrGreen real-time Q-PCR reactions were performed on an Applied Biosystems 7300 Real Time PCR System. The primers used in this study are listed in Table S7.

\section{Supporting Information}

Figure S1 Effect of tcer-1 RNAi on TCER-1::GFP expression. Found at: doi:10.1371/journal.pgen.1000639.s001 (1.37 MB TIF)

Figure S2 Effect of tcer-1 overexpression on the lifespan of germline-precursor (Z2, Z3) ablated worms. 
Found at: doi:10.1371/journal.pgen.1000639.s002 (0.48 MB TIF)

Figure S3 DAF-16/FOXO nuclear localization in germlineablated tcer-1(tm1452) mutants.

Found at: doi:10.1371/journal.pgen.1000639.s003 (0.86 MB TIF)

Figure S4 Expression of F52H3.5 in wild type, $g l p-1$ mutant, and tcer-1; glp-1 mutant backgrounds.

Found at: doi:10.1371/journal.pgen.1000639.s004 (2.08 MB TIF)

Figure S5 Role of tcer-1 in the longevity of mutants in daf-16independent pathways.

Found at: doi:10.1371/journal.pgen.1000639.s005 (0.39 MB TIF)

Table S1 Effect of tcer-1 RNAi and mutation on the lifespans of glp-1 mutants and wild-type worms.

Found at: doi:10.1371/journal.pgen.1000639.s006 (0.15 MB PDF)

Table S2 Effect of tcer-1 overexpression on the lifespan of wildtype animals, tcer- 1 and long-lived mutants.

Found at: doi: 10.1371/journal.pgen.1000639.s007 (0.14 MB PDF)

Table S3 Expression of DAF-16/FOXO-target genes in wildtype animals, $g l p-1$ and $d a f-2$ mutants.

Found at: doi:10.1371/journal.pgen.1000639.s008 (0.15 MB PDF)

Table S4 Effect of daf-16(mu86) and kri-1(ok1251) mutations on lifespan extension induced by tcer-1 overexpression.

Found at: doi:10.1371/journal.pgen.1000639.s009 (0.11 MB PDF)

\section{References}

1. Hsin H, Kenyon C (1999) Signals from the reproductive system regulate the lifespan of C. elegans. Nature 399: 362-366.

2. Arantes-Oliveira N, Apfeld J, Dillin A, Kenyon C (2002) Regulation of life-span by germ-line stem cells in Caenorhabditis elegans. Science 295: 502-505.

3. Yamawaki TM, Arantes-Oliveira N, Berman JR, Zhang P, Kenyon C (2008) Distinct activities of the germline and somatic reproductive tissues in the regulation of Caenorhabditis elegans' longevity. Genetics 178: 513-526.

4. Arantes-Oliveira N, Berman JR, Kenyon G (2003) Healthy animals with extreme longevity. Science 302: 611 .

5. Flatt T, Min KJ, D'Alterio C, Villa-Cuesta E, Cumbers J, et al. (2008) Drosophila germ-line modulation of insulin signaling and lifespan. Proc Natl Acad Sci U S A 105: 6368-6373.

6. Cargill SL, Carey JR, Muller HG, Anderson G (2003) Age of ovary determines remaining life expectancy in old ovariectomized mice. Aging Cell 2: 185-190.

7. Lin K, Dorman JB, Rodan A, Kenyon C (1997) daf-16: An HNF-3/forkhead family member that can function to double the life-span of Caenorhabditis elegans. Science 278: 1319-1322.

8. Lee RY, Hench J, Ruvkun G (2001) Regulation of C. elegans DAF-16 and its human ortholog FKHRL1 by the daf-2 insulin-like signaling pathway. Curr Biol 11: $1950-1957$.

9. Henderson ST, Johnson TE (2001) daf-16 integrates developmental and environmental inputs to mediate aging in the nematode Caenorhabditis elegans. Curr Biol 11: 1975-1980.

10. Antebi A, Yeh WH, Tait D, Hedgecock EM, Riddle DL (2000) daf-12 encodes a nuclear receptor that regulates the dauer diapause and developmental age in $C$. elegans. Genes Dev 14: 1512-1527.

11. Lin K, Hsin H, Libina N, Kenyon C (2001) Regulation of the Caenorhabditis elegans longevity protein DAF-16 by insulin/IGF-1 and germline signaling. Nat Genet 28: 139-145.

12. Libina N, Berman JR, Kenyon C (2003) Tissue-specific activities of C. elegans DAF-16 in the regulation of lifespan. Cell 115: 489-502.

13. Berman JR, Kenyon C (2006) Germ-cell loss extends C. elegans life span through regulation of DAF-16 by kri-1 and lipophilic-hormone signaling. Cell 124: $1055-1068$

14. Jia K, Albert PS, Riddle DL (2002) DAF-9, a cytochrome P450 regulating $C$. elegans larval development and adult longevity. Development 129: 221-231.

15. Gerisch B, Weitzel C, Kober-Eisermann C, Rottiers V, Antebi A (2001) A hormonal signaling pathway influencing C. elegans metabolism, reproductive development, and life span. Dev Cell 1: 841-851.

16. Kenyon C, Chang J, Gensch E, Rudner A, Tabtiang R (1993) A C. elegans mutant that lives twice as long as wild type. Nature 366: 461-464.

17. Kenyon C (2005) The plasticity of aging: insights from long-lived mutants. Cell 120: $449-460$.

18. Burgering BM (2008) A brief introduction to FOXOlogy. Oncogene 27: 2258-2262.

19. Paradis S, Ruvkun G (1998) Caenorhabditis elegans Akt/PKB transduces insulin receptor-like signals from AGE-1 PI3 kinase to the DAF-16 transcription factor. Genes Dev 12: 2488-2498.
Table S5 Effect of tcer-1 reduction of function on the lifespans of other long-lived mutants.

Found at: doi:10.1371/journal.pgen.1000639.s010 (0.11 MB PDF)

Table S6 Strains used in this study.

Found at: doi:10.1371/journal.pgen.1000639.s011 (0.12 MB PDF)

Table S7 Q-PCR primers used in this study.

Found at: doi:10.1371/journal.pgen.1000639.s012 (0.07 MB PDF)

\section{Acknowledgments}

We are grateful to Shohei Mitani (National Bioresource Project, Tokyo, Japan), David Baillie (C. elegans Gene Expression Consortium funded by Genome British Columbia and Genome Canada) and the Caenorhabditis Genetics Center (supported by the National Institutes of Health- National Center for Research Resources) for providing many of the strains used in this study. Our thanks to S. J. Lee, M. Gaglia, M. Suchanek, A. Kao, D. David, and J. Berman for providing valuable comments on this manuscript, and to members of the Kenyon Lab for advice and support. We are thankful to P. Zhang and M. Cary for reagents and analyses.

\section{Author Contributions}

Conceived and designed the experiments: AG CK. Performed the experiments: AG SHK. Analyzed the data: AG CK. Wrote the paper: AG CK.

20. Paradis S, Ailion M, Toker A, Thomas JH, Ruvkun G (1999) A PDK1 homolog is necessary and sufficient to transduce AGE-1 PI3 kinase signals that regulate diapause in Caenorhabditis elegans. Genes Dev 13: 1438-1452.

21. Hertweck M, Gobel G, Baumeister R (2004) C. elegans SGK-1 is the critical component in the Akt/PKB kinase complex to control stress response and life span. Dev Cell 6: 577-588.

22. Oh SW, Mukhopadhyay A, Dixit BL, Raha T, Green MR, Tissenbaums HA (2006) Identification of direct DAF-16 targets controlling longevity, metabolism and diapause by chromatin immunoprecipitation. Nat Genet 38: 251-257.

23. Murphy CT, McCarroll SA, Bargmann CI, Fraser A, Kamath RS, et al. (2003) Genes that act downstream of DAF-16 to influence the lifespan of Caenorhabditis elegans. Nature 424: 277-283.

24. Murphy CT (2006) The search for DAF-16/FOXO transcriptional targets: approaches and discoveries. Exp Gerontol 41: 910-921.

25. McElwee J, Bubb K, Thomas JH (2003) Transcriptional outputs of the Caenorhabditis elegans forkhead protein DAF-16. Aging Cell 2: 111-121.

26. Tatar M, Kopelman A, Epstein D, Tu MP, Yin CM, et al. (2001) A mutant Drosophila insulin receptor homolog that extends life-span and impairs neuroendocrine function. Science 292: 107-110.

27. Holzenberger M, Dupont J, Ducos B, Leneuve P, Geloen A, et al. (2003) IGF-1 receptor regulates lifespan and resistance to oxidative stress in mice. Nature 421: 182-187.

28. Willcox BJ, Donlon TA, He Q, Chen R, Grove JS, et al. (2008) FOXO3A genotype is strongly associated with human longevity. Proc Natl Acad Sci U S A 105: 13987-13992.

29. Pawlikowska L, Hu D, Huntsman S, Sung A, Chu C, et al. (2009) Association of common genetic variation in the insulin/IGF1 signaling pathway with human longevity. Aging Cell. In press. doi: 10.1111/j.1474-9726.2009.00493.x.

30. Kuningas M, Magi R, Westendorp RG, Slagboom PE, Remm M, et al. (2007) Haplotypes in the human Foxola and Foxo3a genes; impact on disease and mortality at old age. Eur J Hum Genet 15: 294-301.

31. Flachsbart F, Caliebe A, Kleindorp R, Blanche H, von Eller-Eberstein $\mathrm{H}$, et al (2009) Association of FOXO3A variation with human longevity confirmed in German centenarians. Proc Natl Acad Sci U S A 106: 2700-2705.

32. Anselmi CV, Malovini A, Roncarati R, Novelli V, Villa F, et al. (2009) Association of the FOXO3A locus with extreme longevity in a southern italian centenarian study. Rejuvenation Res 12: 95-104.

33. Suh Y, Atzmon G, Cho MO, Hwang D, Liu B, et al. (2008) Functionally significant insulin-like growth factor I receptor mutations in centenarians. Proc Natl Acad Sci U S A 105: 3438-3442.

34. Austin J, Kimble J (1987) $g l p-1$ is required in the germ line for regulation of the decision between mitosis and meiosis in C. elegans. Cell 51: 589-599.

35. Sune C, Hayashi T, Liu Y, Lane WS, Young RA, et al. (1997) CA150, a nuclear protein associated with the RNA polymerase II holoenzyme, is involved in Tatactivated human immunodeficiency virus type 1 transcription. Mol Cell Biol 17: 6029-6039. 
36. Sune C, Garcia-Blanco MA (1999) Transcriptional cofactor CA150 regulates RNA polymerase II elongation in a TATA-box-dependent manner. Mol Cell Biol 19: 4719-4728.

37. Holbert S, Denghien I, Kiechle T, Rosenblatt A, Wellington C, et al. (2001) The Gln-Ala repeat transcriptional activator CA150 interacts with huntingtin: neuropathologic and genetic evidence for a role in Huntington's disease pathogenesis. Proc Natl Acad Sci U S A 98: 1811-1816.

38. McKay SJ, Johnsen R, Khattra J, Asano J, Baillie DL, et al. (2003) Gene expression profiling of cells, tissues, and developmental stages of the nematode $C$. elegans. Cold Spring Harb Symp Quant Biol 68: 159-169.

39. Larsen PL, Albert PS, Riddle DL (1995) Genes that regulate both development and longevity in Caenorhabditis elegans. Genetics 139: 1567-1583.

40. Lakowski B, Hekimi S (1998) The genetics of caloric restriction in Caenorhabditis elegans. Proc Natl Acad Sci U S A 95: 13091-13096.

41. Lee SS, Lee RY, Fraser AG, Kamath RS, Ahringer J, et al. (2003) A systematic RNAi screen identifies a critical role for mitochondria in C. elegans longevity. Nat Genet 33: 40-48.

42. Feng J, Bussiere F, Hekimi S (2001) Mitochondrial electron transport is a key determinant of life span in Caenorhabditis elegans. Dev Cell 1: 633-644.

43. Dillin A, Hsu AL, Arantes-Oliveira N, Lehrer-Graiwer J, Hsin H, et al. (2002) Rates of behavior and aging specified by mitochondrial function during development. Science 298: 2398-2401.

44. Salih DA, Brunet A (2008) FoxO transcription factors in the maintenance of cellular homeostasis during aging. Curr Opin Cell Biol 20: 126-136.

45. Tissenbaum HA, Guarente L (2001) Increased dosage of a sir-2 gene extends lifespan in Caenorhabditis elegans. Nature 410: 227-230.

46. Hsu AL, Murphy CT, Kenyon C (2003) Regulation of aging and age-related disease by DAF-16 and heat-shock factor. Science 300: 1142-1145.

47. Greer EL, Dowlatshahi D, Banko MR, Villen J, Hoang K, et al. (2007) An AMPK-FOXO pathway mediates longevity induced by a novel method of dietary restriction in C. elegans. Curr Biol 17: 1646-1656.

48. Oh SW, Mukhopadhyay A, Svrzikapa N, Jiang F, Davis RJ, et al. (2005) JNK regulates lifespan in Caenorhabditis elegans by modulating nuclear translocation of forkhead transcription factor/DAF-16. Proc Natl Acad Sci U S A 102: $4494-4499$.

49. Goldstrohm AC, Albrecht TR, Sune C, Bedford MT, Garcia-Blanco MA (2001) The transcription elongation factor CA150 interacts with RNA polymerase II and the pre-mRNA splicing factor SF1. Mol Cell Biol 21: 7617-7628.

50. Carty SM, Goldstrohm AC, Sune C, Garcia-Blanco MA, Greenleaf AL (2000) Protein-interaction modules that organize nuclear function: $\mathrm{FF}$ domains of CA150 bind the phosphoCTD of RNA polymerase II. Proc Natl Acad Sci U S A 97: 9015-9020.
51. Smith MJ, Kulkarni S, Pawson T (2004) FF domains of CA150 bind transcription and splicing factors through multiple weak interactions. Mol Cell Biol 24: 9274-9285.

52. Pearson JL, Robinson TJ, Munoz MJ, Kornblihtt AR, Garcia-Blanco MA (2008) Identification of the cellular targets of the transcription factor TCERG1 reveals a prevalent role in mRNA processing. J Biol Chem 283: 7949-7961.

53. Lin KT, Lu RM, Tarn WY (2004) The WW domain-containing proteins interact with the early spliceosome and participate in pre-mRNA splicing in vivo. Mol Cell Biol 24: 9176-9185.

54. Proft M, Mas G, de Nadal E, Vendrell A, Noriega N, et al. (2006) The stressactivated $\mathrm{Hog} 1$ kinase is a selective transcriptional elongation factor for genes responding to osmotic stress. Mol Cell 23: 241-250.

55. Nojima M, Huang Y, Tyagi M, Kao HY, Fujinaga K (2008) The Positive Transcription Elongation Factor b Is an Essential Cofactor for the Activation of Transcription by Myocyte Enhancer Factor 2. J Mol Biol Oct 3; 382: 275-87. Epub 2008 Jul 16.

56. Huang H, Tindall DJ (2007) Dynamic FoxO transcription factors. J Cell Sci 120: 2479-2487.

57. Calnan DR, Brunet A (2008) The FoxO code. Oncogene 27: 2276-2288.

58. Wang MC, O'Rourke EJ, Ruvkun G (2008) Fat metabolism links germline stem cells and longevity in C. elegans. Science 322: 957-960.

59. van Heemst D, Beekman M, Mooijaart SP, Heijmans BT, Bradt BW, et al. (2005) Reduced insulin/IGF-1 signalling and human longevity. Aging Cell 4: 79-85.

60. Sutter NB, Bustamante CD, Chase K, Gray MM, Zhao K, et al. (2007) A single IGF1 allele is a major determinant of small size in dogs. Science 316: 112-115.

61. Brenner S (1974) The genetics of Caenorhabditis elegans. Genetics 77: 71-94.

62. Hobert O (2002) PCR fusion-based approach to create reporter gene constructs for expression analysis in transgenic C. elegans. Biotechniques 32: 728-730.

63. Mello C, Fire A (1995) DNA transformation. Methods Cell Biol 48: 451-482.

64. Hansen M, Hsu AL, Dillin A, Kenyon G (2005) New genes tied to endocrine, metabolic, and dietary regulation of lifespan from a Caenorhabditis elegans genomic RNAi screen. PLoS Genet 1: e17. doi:10.1371/journal.pgen.0010017.

65. Lamesch P, Milstein S, Hao T, Rosenberg J, Li N, et al. (2004) C. elegans ORFeome version 3.1: increasing the coverage of ORFeome resources with improved gene predictions. Genome Res 14: 2064-2069.

66. Kamath RS, Fraser AG, Dong Y, Poulin G, Durbin R, et al. (2003) Systematic functional analysis of the Caenorhabditis elegans genome using RNAi. Nature 421: 231-237.

67. Austin J, Kenyon C (1994) Cell contact regulates neuroblast formation in the Caenorhabditis elegans lateral epidermis. Development 120: 313-323.

68. Tullet JM, Hertweck M, An JH, Baker J, Hwang JY, et al. (2008) Direct inhibition of the longevity-promoting factor SKN-1 by insulin-like signaling in $C$. elegans. Cell 132: 1025-1038. 